Revista 2020

\title{
Modernización de la educación virtual y su incidencia en el contexto de las Tecnologías de la Información y la Comunicación (TIC) ${ }^{*}$
}

\author{
Ernesto Fajardo Pascagaza ${ }^{a}$ Luis Carlos Cervantes Estrada
}

\begin{abstract}
Resumen: El presente artículo busca realizar un ejercicio reflexivo en torno a la modernización de la educación virtual y su incidencia en el contexto de las Tecnologías de la Información y la Comunicación (TIC). Para tal efecto, se presenta un ideario temático teniendo como referente, en primer lugar, la modernización de la educación en Colombia en tanto aporte de la virtualidad. En un segundo apartado, se aborda reflexivamente la necesidad de una reforma educativa en la era digital en tanto que la educación necesita un cambio dada la influencia de las TIC y especialmente de internet. Finalmente, se plantean retos y posibilidades que tienen las TIC para abordar los procesos educativos.
\end{abstract}

Palabras clave: educación virtual; TIC; virtualidad; modernización; reforma educativa.

Recibido: 13 de abril de 2020. Aceptado: 19 de junio de 2020.

Disponible en línea: 22 de octubre de 2020.

Cómo citar: Fajardo Pascagaza, E. y Cervantes Estrada, L. C. (2020). Modernización de la educación virtual y su incidencia en el contexto de las tecnologías de la información y la comunicación. Academia y Virtualidad, 13(2), 103-116. https://doi.org/10.18359/ravi.4724

* Artículo de investigación. Investigación realizada en el Grupo ECSAN de la Escuela de Cadetes de Policía General Francisco de Paula Santander.

a Magíster en Educación y en Filosofía. Profesor e investigador de la Escuela de Cadetes de Policía General Francisco de Paula Santander, Bogotá, Colombia. Correo electrónico: ernestofajardo@usantotomas.edu.co ORCID: https://orcid.org/0000-0003-1168-9512

b Magíster en docencia e investigación universitaria. Escuela de Cadetes de Policía General Francisco de Paula Santander, Bogotá, Colombia. Correo electrónico: luiscervantes@policia.gov.co ORCID: https://orcid.org/0000-0002-5706-3251 


\title{
Modernization of virtual education and its incidence in the context of Information and Communication Technologies (ICT)
}

\begin{abstract}
This article intends to carry out a reflective exercise around the modernization of virtual education and its incidence in the context of Information and Communication Technologies (ICT). For such purpose, a group of ideas is presented, first taking as a referent the modernization of education in Colombia as a contribution of virtuality. In a second section, the need for an educational reform in the digital age is reflexively addressed, as education needs a change given the influence of ICTs and especially the internet. Finally, there are challenges and possibilities that ICTs face in order to address educational processes.
\end{abstract}

Keywords: virtual education; ICT; virtuality; modernization; education reform.

\section{Modernização da educação virtual e sua incidência no contexto das Tecnologias da Informação e Comunicação (TIC)}

Resumem: O presente artigo procura realizar um exercício reflexivo sobre a modernização da educação virtual e sua incidência no contexto das Tecnologias da Informação e Comunicação (TIC). Para tal efeito, apresenta-se um ideário temático que tem como referente, em primeiro lugar, a modernização da educação na Colômbia como contribuição da virtualidade. Num segundo momento, aborda-se reflexivamente a necessidade de uma reforma educativa na era digital já que a educação precisa de uma mudança devido à influência das TIC e especialmente da internet. Finalmente, propõem-se desafios e possibilidades que as TIC têm para abordar os processos educativos.

Palavras-chave: educação virtual; TIC; virtualidade; modernização; reforma educativa. 


\section{Introducción}

Es necesario ahondar en la influencia que tiene la educación en el desarrollo de la sociedad y como esta permite fortalecer sus cimientos para adaptarse a nuevos fenómenos, cambios y circunstancias propias de la constante evolución en la que el ser humano está inmerso y que no puede evadir. Por lo tanto, es oportuno reconocer la relación existente entre la educación y la revolución tecnológica de la comunicación e información, a partir del análisis y surgimiento de las nuevas transformaciones que se han dado en los patrones tradicionales y de todos los factores que potencializaron el acceso a la información, generando consigo un mayor bienestar y progreso en el conglomerado social.

En este sentido, es pertinente realizar un ejercicio reflexivo en torno a la modernización de la educación virtual y su incidencia en el contexto de las TIC. En clave de la modernización global, la educación juega un papel fundamental y las TIC son herramientas favorecedoras de los procesos de enseñanza aprendizaje. Los sistemas de gobierno han de propender por un sano presupuesto en aras de invertir en plataformas virtuales que favorezcan la educación de las personas sin exclusión, sino al contrario, facilitando los mejores recursos de tal forma que se optimicen los procesos y los resultados educativos.

Las nuevas tecnologías han de estar al servicio de la educación para disipar las brechas sociales que se han generado por la falta de recursos económicos, logísticos y de cultura digital. La tecnología abre una nueva puerta para el conocimiento y a la facilidad de acceso al conocimiento de la historia de las naciones, así como a los saberes de las diferentes áreas.

La modernización de la educación virtual plantea la necesidad de generar reformas educativas en el contexto de la era digital a partir de la influencia de las TIC. En este sentido, es necesario reconocer que el acceso a las TIC trae ventajas y desventajas en el ejercicio educativo. Para muchas personas dadas sus circunstancias locativas y de conectividad, su acceso se imposibilita en la práctica, como es el caso de quienes habitan en zonas rurales, sin embargo, hay personas que pueden beneficiarse significativamente de las plataformas virtuales porque su acceso y conectividad les permite navegar con facilidad de escenarios citadinos.

El uso adecuado de las TIC en la educación abre nuevos horizontes de comprensión del quehacer educativo y su validez en la apropiación del conocimiento y, por lo tanto, genera nuevos retos y posibilidades de implementación de las TIC en el contexto de las instituciones educativas tanto privadas como públicas a la hora de utilizar plataformas que respondan a las necesidades de sus usuarios en condiciones de equidad social. En este sentido, es necesario que los gobiernos y las instituciones educativas presupuesten invertir en tecnologías de avanzada para responder a la modernización de la educación virtual y sus contextos emergentes.

\section{Modernización de la educación en Colombia y su aporte desde la virtualidad}

En la historia de la humanidad se han presentado diferentes etapas o momentos que representan un cambio en la vida y contexto de cada uno de los seres humanos. Desde el inicio de los tiempos el ser humano ha estado en constante evolución, lo que lo ha llevado a buscar y dar solución a diferentes problemas que se han presentado o cuestionarse sobre cada uno de los acontecimientos diarios que lo rodean y complementan. Esto a su vez, ha generado una reestructuración en el pensamiento, en las creencias e ideas de cada uno de los individuos que hacen parte de su entorno, estudiando los acontecimientos de su cotidianidad y asimilando procesos de aprendizaje en la construcción de los saberes especializados. Gracias a este constante desarrollo e inquietud por el conocimiento se ha desarrollado la sociedad, hasta llegar al punto en el que se conoce hoy en día bajo la impronta de la sociedad del conocimiento.

En este nuevo siglo el hombre se ha caracterizado por entrar a una nueva era, la era digital, la cual se ha convertido en una realidad planetaria causando que el hombre cambie su devenir cotidiano a partir de la incursión de los avances tecnológicos y la consiguiente afectación de los 
diferentes contextos que lo rodean, en especial, el contexto pedagógico y cómo este componente interactúa con las nuevas generaciones y el sentido de una modernización en la educación, reconociendo que ofrece facilidades, pero a su vez, retos que deben asumirse para seguir avanzando. En la actualidad se vive en permanente incertidumbre, debido a que no se tiene un conocimiento claro sobre este nuevo componente tecnológico y no se hace un buen uso de este, lo que ocasiona diversos problemas, los cuales, a pesar de contar con características similares a otros, no pueden ser solucionados de igual forma, por lo que se requieren respuestas diferentes a las planteadas antes (Balart y Cortés, 2018).

Se hace necesario que la incorporación de las herramientas tecnológicas a la enseñanza se haga a partir de un nuevo concepto de alfabetización y surge de la necesidad de la actualización constante a la que son sometidos los conocimientos o campos del saber. Es fundamental reconocer que el contexto educativo también ha evolucionado en el último siglo, partiendo del concepto de alumno, el cual se fundamentaba en la acumulación y reproducción de información, sin cuestionar nada y llegando al concepto de estudiante, el cual refiere a un ser inteligente capaz de buscar, procesar y comunicar la información que obtiene.

La educación es un componente fundamental para el desarrollo de una sociedad en sus diferentes ámbitos, y el desafío que debe asumir la sociedad y el gobierno es combinar la tecnología con la educación y la cultura de las personas sin dejar de lado conceptos como el humanismo y la equidad social (González, 2001).

A lo largo de la historia se observa que los grandes avances en términos pedagógicos se han producido cuando se ha ocasionado un cambio tecnológico, pero ello no significa que estos cambios hayan sido fáciles ni mucho menos que el hombre haya tenido un pensamiento abierto respecto a ellos. Por el contrario, dichos cambios han llevado a un rechazo por parte de algunos y, así mismo, una aceptación e interiorización en otros lugares (Facundo, 2004).

Dado lo anterior, las nuevas tecnologías son entendidas como un apoyo que puede utilizar el docente, pero no se debe definir como la única herramienta, puesto que hay variables pedagógicas que siguen siendo oportunas y validas en el quehacer de la docencia, como puede ser, en algunos casos, la adquisición de habilidades motrices y experiencias reales que contribuyen en el debido proceso de aprendizaje de cada campo. El docente es entonces el responsable de mediar la utilización de las diferentes herramientas pedagógicas, incorporando ahora la utilización de las TIC (Fajardo, 2019).

Aunque también se presenta un uso de estas herramientas por parte de los estudiantes y la sociedad en general en el que se involucra el ejercicio del autocontrol proveniente del valor de la autonomía. Este valor puede ser adoptado o no por cada persona y esto se ve mayormente reflejado en las instituciones de educación superior, donde se busca homogenizar las condiciones de aprendizaje y beneficiar a aquellos estudiantes reflexivos, críticos, colaborativos y autónomos (Balart y Cortés, 2018).

Ahora bien, desde las instituciones educativas se perciben obstáculos que no han sido solucionados y por lo tanto, afectan la realidad social matizada por el fenómeno de la desigualdad económica y las consecuentes divisiones y clases sociales, lo cual conlleva a que solo quienes tienen el poder del capital podrán acceder al conocimiento, creando exclusión en la adquisición del conocimiento y su incidencia en la educación, dando lugar a la necesidad de contar con instrumentos tecnológicos adecuados para responder con idoneidad a las propuestas educativas y de aprendizaje según los contextos que la sociedad exija. Se genera una dependencia hacia las tecnologías emergentes, lo que podría volverse un problema para el hombre si no se prevén los diversos escenarios para la labor educativa. Esta brecha digital surge entre las personas que pueden incluirse en la adquisición de los equipos y quienes no, lo que se resume en una limitante para algunos y una nueva posibilidad para otros (Fajardo, 2019).

Tomando en consideración lo anterior, el Estado interviene en una serie de políticas y normas que regulan el manejo de la tecnología en la educación, políticas que no pueden ser sectoriales, 
pues lo que se busca es fortalecer el proyecto de país, por lo cual se requiere el compromiso de toda la jerarquía política y en especial, la Presidencia de la Nación. (Lugo y Kelly, 2010). En Colombia, se cuenta con una participación significativa de diversas organizaciones sociales, como son las universidades en tanto que son abanderadas de la educación superior, (Ministerio de Educación Nacional, 2017), en el desarrollo e implementación de las TIC y a su vez en el diseño y próxima ejecución de políticas públicas que se incorporan a los planes del Estado, destacando la importancia de estas y de las bibliotecas, centros y organizaciones culturales que desarrollan redes que fortalecen el intercambio de ideas y la concertación de proyectos entre las diversas instituciones del Estado (Barón y Gómez, 2012).

El desafío sigue siendo el mismo y es cómo involucrar la población que no cuenta con la infraestructura apropiada para implementar las TIC, dado que Colombia es un país subdesarrollado y gran parte de su población se encuentra en un estado de pobreza y vulnerabilidad social. Estos sectores poblacionales no cuentan con las medidas básicas de saneamiento, lo que hace aún más compleja la presencia de un buen sistema de conectividad e interconexión al servicio de internet, estableciendo una división sectorial, pues en algunos sectores no es posible implementar estas herramientas tecnológicas si no se plantean soluciones radicales a los problemas sociales más sobresalientes y su relación próxima con los procesos de enseñanza aprendizaje contextualizados.

En Colombia se están desarrollando medidas para la incorporación de las TIC en el proceso educativo de cada uno de los diferentes escenarios pedagógicos, sin dejar de lado otras variables significativas que no pueden ser reemplazadas por las herramientas de mediación tecnológica, para lograr un buen uso de estas, a partir de la conciencia autónoma por parte de la ciudadanía y la promoción un pensamiento responsable de su uso en la sociedad.

Además, en el país se evidencia un ambiente de precariedad histórica en el funcionamiento adecuado de los sistemas de enseñanza motivado por el fenómeno de la desigualdad social, por el incremento del índice de pobreza y de exclusión, (Maza, Vergara y Román, 2017), lo cual hace más amplia la brecha social. Ante este fenómeno social, resulta de vital importancia que el Estado colombiano se enfoque con mayor esfuerzo en resolver diferentes problemáticas sociales y económicas, las cuales afectan directamente el proceso de desarrollo tanto regional como nacional y afectan diferentes sectores, como el educativo. Ello ocasiona que esta brecha social se incremente con el paso del tiempo y lleve a un retraso en el avance económico y cultural de la misma nación sin poderlo evitar, así se generen políticas que promuevan el uso de las TIC que paradójicamente no tendrán validez sobremanera en las regiones más vulnerables.

La educación virtual es vista por la mayoría de las personas como una oportunidad de crecimiento tanto a nivel personal, laboral y profesional, ya que se puede estudiar desde cualquier lugar del mundo, se puede interactuar con personas de todos los países, aprender nuevos idiomas, compartir conocimiento, en fin, son muchas las ventajas que se le encuentran a esta metodología educativa.

Paras (2017) afirma que: "sería una grave irresponsabilidad histórica no asimilar que los aportes de la revolución tecnológica representan una extraordinaria oportunidad para avanzar en el perfeccionamiento de los sistemas educativos y en la democratización de la información y del conocimiento" (p. 36).

Y es que con los avances en la tecnología se han creado software, plataformas, herramientas que hacen que en la educación virtual y el aprendizaje de los estudiantes sea más interactivo y dinámico y se perciba mejor que la educación presencial en la cual se tiene una rutina establecida. En este sentido, Becerra (2017) afirma que:

uno de los mayores atributos de los modelos virtuales es que rompen las barreras geográficas, cada persona desde su lugar de residencia sin necesidad de desplazarse, puede asumir su propia capacitación, satisfacer sus necesidades formativas y mejorar su calidad de vida a través de un programa de educación virtual (p. 58). 
Para Santiago (2001) hay una evidente paradoja en el contexto de la educación, porque, por una parte, ha sido transmisora de conocimientos y saberes de generación en generación en contextos de encuentros sociales personales, pero hoy, por otra parte, se ve abocada a responder a los nuevos modelos y paradigmas que la modernidad exige, es decir, la virtualidad funcional. En este sentido, para Munevar (2017) las políticas educativas han de propender por una educación de calidad formando en el recurso humano y, por lo tanto, garantizando una obra de mano calificada al servicio de la sociedad y la competitividad en escenarios de presencialidad y virtualidad.

Se presenta una relación entre lo que vive la sociedad actual y la educación virtual permitiendo destacar, en medio de la propagación del COVID19, la necesidad de tomar medidas preventivas como el aislamiento en casa por un largo periodo de tiempo y por consiguiente dejar de asistir a las clases presenciales como se tenía acostumbrado, dando lugar a la educación remota mediada por herramientas virtuales y así evitar que los estudiantes pierdan clases. En el momento se tomó como una oportunidad y solo se vieron las ventajas que esta educación traería, pero no se pensó de manera más pertinente en los estudiantes sin recursos económicos para solventar esta eventualidad.

No todo es perfecto y por lo tanto se presentan desventajas que esta modalidad educativa trae a la población que incursiona en procesos de educabilidad. Para González (2017) hay estudiantes que no tienen el sentido de la autonomía y la independencia y, por otra parte, es importante considerar la necesidad de las prácticas reales como método pertinente de aprendizaje que el modelo virtual no puede suplir.

Como bien se sabe, hay estudiantes que tienen problemas de aprendizaje y necesitan de una persona que los oriente. Muchos niños son hijos de personas con escasos recursos económicos que para poder sobrevivir y conseguir el sustento diario deben ir a trabajar y no tienen tiempo de cuidar a sus hijos y el colegio, en estos casos, no solo sirve para que los niños se eduquen y aprendan, sino también como un lugar en el que los niños están seguros. Ahora bien, hay otros casos en los que en el colegio se les brinda a los niños el refrigerio y el almuerzo y en la casa los padres no asumen esta responsabilidad porque sus ingresos no son suficientes para suplir estos gastos.

Las clases virtuales no ofrecen estos beneficios que son significativos para esas familias humildes $\mathrm{y}$ vulnerables $\mathrm{y}$ en este punto cabe preguntarse: ¿qué pasará con estos niños?, ¿los padres podrán ir a trabajar tranquilos, sabiendo que sus hijos quedan solos en casa aguantando hambre?, ¿qué pasará con los estudiantes que no tienen un computador con los mínimos para acceder a plataformas virtuales y estar en igualdad de condiciones como los niños que tienen todos los recursos y ayudas de última generación tecnológica?

Mientras los estudiantes con estos beneficios aprenden y van avanzando en los temas del año escolar, los estudiantes menos favorecidos se quedan rezagados y su año escolar se ve gravemente afectado. No se trata de estar en contra de la educación virtual, pero sí de revisar que las medidas tomadas hayan sido estudiadas adecuadamente, pues es claro que un gran número de estudiantes no cuenta con un computador, un celular, acceso a internet en su casa, y, es más, en algunas zonas del país ni siquiera tienen luz, dificultando aún más la educación virtual.

Con el pasar de los años estos niños y jóvenes que no se han podido preparar de la misma manera que sus compañeros que si lo pueden hacer, se van a enfrentar a unas pruebas que definen su conocimiento, salen los resultados y Colombia queda entre los últimos puestos; es ahí donde se van a ver los grandes vacíos que los estudiantes han tenido a lo largo de su vida escolar por el hecho de no tener acceso a estas herramientas virtuales como se confirma en estudios actuales sobre esta problemática en el país (Acevedo y Villamizar, 2018).

Y como siempre, las consecuencias recaen en las personas más vulnerables y todos los entes responsables sacan miles de excusas para justificar sus fallas: una de ellas es decir que ¡los estudiantes son los responsables que Colombia se situé entre los países con los resultados más bajos respecto a los promedios de otros países! ¡que cómo no van a estudiar si es que ahora todo es más fácil y con la tecnología el que no aprende es porque no quiere! 
Esto es cierto, pero no todos tienen los mismos recursos y facilidades para estudiar de manera virtual.

Otra desventaja que aflora es que no todos los niños tienen padres responsables y a algunos no les importa en lo más mínimo que sus hijos se eduquen; por el contrario, ven las clases virtuales como una pérdida de tiempo y obligan a sus hijos a hacer los oficios, a que trabajen y poco a poco los niños le pierden el interés por el estudio y más si tienen unos padres que no se preocupan porque sus hijos tengan acceso a este tipo de actividades de aprendizaje virtual.

Otros estudiantes tienen problemas de depresión y trastornos de personalidad y estar encerrados tanto tiempo no les ayuda en nada, en algunas ocasiones porque no entienden las tareas que deben realizar y esto hace que sientan ansiedad y deseen estudiar presencialmente, pues en los centros educativos pueden interactuar con sus compañeros, jugar, pedir a sus profesores que les aclaren las dudas que tienen y olvidar por un momento los problemas que viven cotidianamente en sus hogares.

Aunado a lo anterior, según Contreras (2018) en la década de los ochenta el modelo de educación a distancia favoreció muchos procesos de aprendizaje, sin embargo, por el anhelo de tener más cobertura en los programas, no se consideraron aspectos fundamentales como la formación de los tutores, la calidad del material didáctico y de enseñanza, puesto que era un material único para todos y no se consideraban las diferencias regionales como contextos propios de aprendizaje. Se ha llegado a pensar que aprender a distancia y virtualmente es fácil y no requiere demasiado tiempo, no obstante, este argumento pierde peso ya que es más exigente dadas las consideraciones del trabajo autónomo y disciplinado.

Otra desventaja, no menos importante de las clases virtuales, es que afectan la salud de las personas. Los estudiantes deben pasar varias horas del día frente a su computador y si no tienen la costumbre de hacer descansos y pausas activas con el tiempo pueden presentar problemas visuales, musculares, por movimientos repetitivos y malas posturas ergonómicas. Además, no todos los estudiantes tienen un lugar cómodo donde estudiar, lo que significa que el deterioro de su salud puede ser mayor.

Analizando todos estos aspectos, cabe resaltar que el país no está preparado para enfrentar una educación virtual al ciento por ciento, porque, aunque son más los beneficios que las desventajas, faltan muchas variables para que la educación virtual logre ser mejor que la presencial. Esto exige capacitación y entrenamiento para manejar todas las herramientas que ofrece la educación virtual $\mathrm{y}$, por consiguiente, cerrar las brechas que se han abierto por la falta de recursos económicos en los estudiantes más pobres y su acceso a esta modalidad de aprendizaje.

Hablar desde la perspectiva de la modernización implica comprender que las TIC han experimentado cambios significativos a partir de los procesos de interconexión sujetos a la red, potencializando aplicaciones, empleando nuevas didácticas, replanteando nuevos conocimientos y habilidades tanto de los docentes como de los estudiantes sobre la manera adecuada de enseñar en contextos y escenarios virtuales.

Se trata de un fenómeno educativo reciente que invita a los docentes a cambiar sus prácticas educativas frente a los nuevos paradigmas aprovechando las tecnologías emergentes para la creación de nuevos ambientes y dinámicas de enseñanza y aprendizaje incorporando escenarios flexibles según los momentos y los contextos educativos.

\section{Necesidad de una reforma educativa en la era digital}

En esta época, la sociedad ha tenido múltiples cambios en diversos escenarios impulsados mayoritariamente por los avances en la información y la tecnología. Sin embargo, hay situaciones que pese a que han cambiado y mejorado se siguen presentando sin grandes diferencias en el contexto de la educación, especialmente en países que están en vía de desarrollo, porque no se ha podido garantizar una educación básica para toda la población, vulnerando así uno de los derechos primordiales de toda persona, como el derecho a la educación. 
No obstante, la sociedad ha venido evolucionando y este tipo de situaciones ha ido disminuyendo considerablemente. Esto se puede atribuir a diferentes factores, entre ellos, la información, la tecnología y su avance exponencial. La sociedad ha evolucionado a partir de la injerencia de las múltiples posibilidades y aplicaciones que se han podido hacer con el uso de la información y la tecnología. Tal es el caso de la implementación de internet en los escenarios educativos dados sus beneficios en los procesos de enseñanza aprendizaje y porque: "todo este uso de datos digitales conducirá a procesos educativos más eficientes y transparentes, además de dar soporte al auto seguimiento y la auto diagnosis del aprendizaje por parte de los interesados" (Selwyn, 2013, p. 56).

El uso de internet beneficia a muchas más personas, con su aplicación permite mejores resultados en el aprendizaje de las personas que usan este tipo de estrategias frente a las que no las utilizan y evidencia la superioridad del uso de estrategias relacionadas con el internet frente a una educación que es únicamente presencial. Además, los cambios de este modelo de educación no deberían ser realizados únicamente por los agentes privados según opina González (2001), sino que deberían ser planeados e implementados por el propio Estado.

Uno de los beneficios que trae implementar este tipo de estrategias es el de ayudar a reducir el costo y mantenimiento de la educación en el país, puesto que se ahorrarían gastos de desplazamiento en los que incurren normalmente quienes se desplazan a las instituciones educativas. Además, beneficiaría a las empresas que se basan en el mercado de la tecnología, ya que invertirían más dinero en la venta de sus equipos tecnológicos respondiendo a los estándares de competitividad en el mercado laboral y con esto mejorar mejorar la calidad de vida de quienes se relacionan con el entorno educativo, es decir, estudiantes, docentes y padres de familia.

Por lo tanto, es necesario ahondar en la influencia que tiene la educación en el desarrollo de la sociedad y cómo esta permite fortalecer los cimientos para posteriormente adaptarse a nuevos fenómenos, cambios y circunstancias, resultado de la constante evolución en la que el ser humano está inmerso y no puede evadir. En este sentido, es oportuno reconocer la relación existente entre la educación y la revolución tecnológica de la comunicación e información a partir del análisis del surgimiento de las nuevas transformaciones ocasionadas en los patrones tradicionales y de todos los factores que han potencializado el acceso a la información, lo cual ha traído consigo un mayor bienestar y progreso para el conglomerado social.

La educación ha sido un pilar fundamental en el desarrollo de la sociedad, a partir de los descubrimientos e innovaciones científicas y tecnológicas materializadas en nuevos modelos comunicacionales que "cancelan fronteras y límites en las distancias y en el tiempo" (Paras, 2001, p.24). Ahora bien, el reto que surge de estas transformaciones en el entorno social, político y económico es logar amalgamar el nuevo fenómeno tecnológico con la sociedad sin dejar de lado sus características propias, es decir, el humanismo, la democracia, la equidad y armonía social, facilitando la obtención del conocimiento por medios diferentes a los tradicionales y dando finalmente apertura a la visión de internet como un recurso innovador que fomenta el desarrollo educativo.

En concordancia con lo anterior, el uso generalizado de internet como fuente de conocimiento proporciona ventajas tanto a nivel individual como colectivo y genera cambios notables en el saber humano, porque incentiva a mejorar los métodos de enseñanza y aprendizaje, lo cual a futuro podría llegar a satisfacer completamente una de las necesidades básicas del ser humano que es la educación. Lo anterior es de vital importancia ya que incide directamente en el desarrollo óptimo e integral de las personas.

En efecto, para lograr este salto cualitativo en la educación virtual es de suma importancia generar conciencia en lo relativo a la conectividad existente entre el campo educativo y las tecnologías de las comunicaciones, es decir, verlo como una prioridad para el progreso social y para promover en las nuevas generaciones el uso de estas llegando poco a poco a introducir una connotación de necesidad o de interdependencia. Sumado a esto, al potencializar los sistemas educativos se fortalece el avance y producción del conocimiento 
de la mano con la formación de nuevos sujetos que optimizan el desarrollo de las diferentes disciplinas, ya sean científicas o humanísticas, pero que finalmente practican y desarrollan los seres humanos. Indiscutiblemente la era digital y tecnológica forman parte de la realidad cotidiana de todos los escenarios educativos porque: "educar en tiempos de Internet implica una formación compatible con nuevas formas de entretener, producir, aprender y trabajar, respetando los estilos de cada individuo y comunidad virtual” (Dávila, 2006, p. 26).

En definitiva, es necesario que el uso de las tecnologías de la información tenga como base que todas las personas posean un acceso igualitario a las mismas, sin embargo, para que esto sea posible, se debe remitir a la responsabilidad que tiene el Estado para que "mitigue las diferencias crecientes entre ciudadanos, grupos y regiones de cada país" (Paras, 2001, p.36). Igualmente, se debe considerar que los objetivos y metas propuestas deben alcanzarse mediante la implementación de políticas públicas y de diferentes mecanismos de coordinación, lo cual finalmente se logra optando por un mejor sistema educativo respaldado y garantizado por el Estado, pero fundamentalmente arraigado o perteneciente a la sociedad para que se puedan obtener resultados favorables y duraderos a largo plazo.

Es importante que la sociedad tenga disposición para acoplarse al uso de la tecnología, sea para labores en una empresa, para la educación o cualquier función que se necesite cumplir, ya que se pertenece a la era donde muchas de las relaciones sociales se ejecutan a partir de la virtualidad.

Es evidente la incidencia y funcionabilidad que tienen las TIC en los escenarios educativos dado que posibilitan nuevos horizontes de enseñanza y desarrollo de competencias, sin embargo, su empleo no se ha optimizado debido a la precariedad de las plataformas y la débil y limitada orientación para su debida utilización en escenarios y contextos educativos. Las instituciones educativas cumplen un papel fundamental en el uso y apropiación de los recursos tecnológicos como apoyo significativo a los procesos de aprendizaje, los cuales son de carácter multidimensional en relación con los emergentes paradigmas que se presentan a la labor docente y sus nuevos roles en la configuración de la enseñanza frente al papel del estudiante considerado como nativo digital.

Las tecnologías digitales y los conocimientos aportados en las redes han dado lugar a nuevos modelos de comunicación y aprendizaje originados a partir de nuevas formalidades de enseñanza. En dichos modelos intervienen docentes y estudiantes que desarrollan habilidades y destrezas con el uso de internet y la producción de nuevos conocimientos y experiencias y eliminan las brechas digitales generacionales; a esta realidad ha de apuntar la reforma educativa.

\section{Retos y posibilidades de las TIC en la educación}

Las TIC son tecnologías o prácticas desarrolladas a partir de los nuevos paradigmas tecnológicos que han sido cada vez más rápidos y cambiantes en las últimas décadas afectando positiva y negativamente el devenir de los seres humanos. Con las TIC se ha podido descubrir un nuevo mundo modificando las técnicas y modalidades de aprendizaje a nivel educativo y a su vez estas han permitido mejorar las áreas científicas y prácticas de las dinámicas de aprendizaje. Las herramientas tecnológicas han evolucionado de manera vertiginosa y han dado lugar a que la educación tenga que asumir nuevos retos en la implementación y adaptación contextualizada de estas mediaciones en los diversos escenarios educativos (Herrera, 2015).

La sociedad actual es una sociedad dinámica, es decir, a medida que pasan los años las personas van cambiando sus hábitos o costumbres generando así un aumento en la necesidad del consumo, porque la misma sociedad se ha encargado en definir unos "estereotipos" dados a partir de los medios de comunicación como la televisión en donde a partir de propagandas, telenovelas y comerciales, entre otros, se define que la felicidad depende de algo material: si se tiene el ultimo $i$ Phone, el mejor carro, la mejor ropa, los zapatos más caros, el bolso más caro, se delimita así una barrera tanto social como económica sobre lo que es la felicidad. En este orden de ideas, las TIC pueden generar a su vez aspectos negativos como el aumento del 
consumismo por adquirir nuevas tecnologías que afectan directamente los recursos naturales.

Pero no todo es afectación en relación con las TIC. Estas también generan aspectos positivos para mejorar la detección de fenómenos naturales que pueden ocasionar una emergencia y aumentar la tasa de mortalidad en el país. Un ejemplo de esto, son los equipos que ayudan a la detección anticipada de un temblor logrando disminuir así el impacto que este produzca en el territorio y generar una respuesta de alerta ante cualquier situación. También existen equipos que regulan la contaminación en el aire lo cual ayuda a tomar medidas correctivas anticipadas y genera la disminución de un impacto mayor en años futuros. Estos equipos se crearon debido al avance tecnológico que ha tenido el mundo a través de la innovación de nuevos productos o técnicas. Por lo tanto, si la sociedad no se hubiese adherido a esos cambios, posiblemente los fenómenos naturales tendrían mayor impacto en el planeta.

Las TIC además de ser fundamentales para el ámbito científico también han generado un gran impacto en el ámbito educativo. La educación que se conocía o a la que estaba acostumbrada la sociedad era una educación únicamente presencial en la que el niño iba a su escuela a tomar clases dictadas por su docente y realizaba sus tareas mediante el apoyo de libros que se encontraban en la biblioteca del pueblo. Usualmente se encontraba una biblioteca por pueblo, lo que generaba mayor concentración de estudiantes y mayor dificultad para la búsqueda y comprensión de textos. En algunas instituciones se prestaba el servicio de biblioteca, pero tenían los mismos limites que tenía la biblioteca del pueblo.

La educación que se les brindaba era muy restringida, el aprendizaje era más teórico porque no se tenían estructuras como laboratorios o demás edificaciones para tener un aprendizaje práctico. Algunos alumnos no contaban con computadores $\mathrm{u}$ otros medios que les facilitaran la búsqueda de información y muchos estudiantes tampoco contaban con la ayuda de sus padres para realizar sus trabajos, lo que les afectaba al no lograr entender correctamente las actividades que deberían resolver.
Los estudiantes de esa época tenían menores oportunidades debido al poco avance de la tecnología, ahora, en la actualidad, los estudiantes tienen mayores oportunidades o formas de acceso al aprendizaje; existen diversas páginas web, mayor facilidad para adquirir un computador, mayor facilidad para adquirir internet en casa y se ofrecen diferentes modalidades de estudio porque el avance de la tecnología lo permite. Las instituciones educativas han implementado aulas virtuales para que los estudiantes interactúen con sus tutores y luego puedan enviar sus documentos fruto de su trabajo académico. Es por eso que una persona puede trabajar y estudiar al mismo tiempo.

Las TIC ofrecen la oportunidad de clases virtuales en las que los estudiantes no tienen que salir de sus casas y pueden recibir la educación que fue pagada desde sus hogares. Las TIC proporcionan diferentes plataformas como : Com8s, Schoology, Edmodo, Course Sites By Blackboard, Lectrio, Udemy, entre otras y estas son empleadas por las universidades o colegios según sus necesidades. Además, ofrecen los mismos beneficios a las personas que no pueden llegar a sus trabajos por el mismo motivo, permitiéndoles realizar sus labores de manera responsable bajo la modalidad de teletrabajo, optimizando el tiempo que se demoraban las personas desde su casa al trabajo y favoreciendo a los trabajadores con horarios flexibles. Todo ello permite a su vez a los empresarios realizar conferencias y reuniones ya sean locales, regionales, o en diferentes países mediante el uso de múltiples plataformas visuales.

Las TIC también puede ser utilizadas para fines sociales, es decir, permiten interactuar con personas ubicadas en zonas geográficamente diferentes, interactuar con diferentes culturas, proporcionan diferentes aplicaciones virtuales. La aplicación más conocida es YouTube, gracias a la cual las personas tienen acceso a música gratuita y a una oferta de videos educativos que le permiten a un estudiante cuando, no comprende un tema, buscarlo en la plataforma, ya que hay canales que se dedican a la explicación educativa.

Mediante las TIC se crearon redes sociales como lo son Facebook, Instagram, WhatsApp entre otras para que las personas pueden interactuar con 
amigos, familiares, sus parejas y demás contactos sociales. Cabe resaltar que muchas de estas redes sociales pueden utilizarse de forma ilícita o de forma negativa por personas inescrupulosas buscando afectar de manera íntegra la salud o bienestar de otras personas.

Las TIC brindan muchas oportunidades a las sociedades tanto educativas, científicas y sociales. Pero, así como hay aportes significativos desde la virtualidad, igualmente mediante las redes se realizan estafas, robos y demás delitos. La sociedad es la encargada del buen manejo de estas redes sociales, de la prevención y el control de la información que se les da a terceras personas con información que usualmente se publica en estas. Se debe tener en cuenta por lo tanto que muchas veces las personas se hacen pasar por otras suplantando su identidad con fines ilegales.

En el siglo XXI la educación desde la tecnología se ha cuestionado de diversas maneras, porque el aprendizaje se divide en dos partes: aprendizajes de diversa naturaleza a lo largo de la vida como las experiencias que se van adquiriendo y aprendizajes que buscan adaptarse de manera oportuna y eficaz a las diversas situaciones que los contextos sociales, familiares, laborales y económicos brinden al mundo cambiante.

Los aprendizajes recibidos en los escenarios educativos son aprendizajes de diversa naturaleza, se adquieren a lo largo de vida y van cualificando las historias de los individuos. Además, son aprendizajes que se van adaptando de manera oportuna y eficaz a las diversas situaciones que los contextos sociales, familiares, laborales y económicos brinden al mundo cambiante.

Como las tecnologías de la información y la comunicación son una herramienta con un potencial grandísimo para el aprendizaje, se entiende que tanto el alumno y el maestro tienen que intervenir para que el aprendizaje sea exitoso, es decir, que no solo es responsabilidad del maestro enseñar, porque lo que hace él es abrir un portal al conocimiento y el estudiante tiene que ir más a fondo.

La incorporación de las TIC ha marcado a las instituciones en la forma de enseñar y en el aprendizaje que sus estudiantes van adquiriendo, porque las clases se vuelven aún más dinámicas y lo tradicional se va quedando atrás, por lo tanto, cada docente debe tener su propio sistema de tecnología y enseñanza ya que cada materia es diferente y su forma de hacerla entender también. A los docentes se le presentan nuevos retos y modelos de trabajo que les exigen asumir nuevas prácticas y dinámicas para vivenciar en el aula de clase. (Castellanos, 2013).

Para que se puedan implementar las TIC toda institución educativa debe contar con unos factores y el principal es el de los recursos humanos, pues todos los demás (contenidos, conectividad y tecnología) tienen relación con este. Estos factores deben ser tenidos en cuenta y priorizados por igual para que las TIC sean implementadas de la mejor manera. Para Tapia y León (2016) cuando se incluyen las TIC en los procesos de enseñanza aprendizaje, estas han de ir asociadas a lineamientos referenciales que permitan la toma de decisiones en pro de la calidad educativa.

Por otro lado, el panorama incluyente que tienen las TIC en la sociedad y en la educación depende de qué tan desarrollado esté el país en donde se va a implementar y, en este sentido, la infraestructura tecnológica y de cobertura que tiene en todos los escenarios posibles del contexto educativo para responder a los nuevos retos y paradigmas educativos. Ahora bien, no solamente se trata de infraestructuras tecnológicas y de cobertura, también se trata de la formación en procesos de enseñanza que tengan los docentes frente a los nuevos ciudadanos nativos digitales y sus emergentes compromisos como protagonistas de la sociedad del conocimiento. Se trata de nuevos retos asumidos por los docentes en la coherencia y creatividad pedagógica respondiendo a las nuevas tendencias educativas para hablar lenguajes paralelos digitales de acuerdo con los tiempos modernos que experimenta la sociedad.

Con todo, las TIC como herramientas didácticas han de propender por el mejoramiento de la calidad educativa de los estudiantes sin dejar de lado su formación integral proyectada hacia un mejor servicio a la sociedad. En este sentido, dentro de lo posibilitante que tienen estas tecnologías está el reconocer su importancia, pero más allá de su 
real importancia, está la apropiación que se pueda tener de ellas para humanizar a las personas, para acceder a la construcción de nuevos conocimientos que contribuyan a ser mejores seres humanos, con pensamiento crítico, creativo y ético.

Frente a los nuevos retos y posibilidades de las TIC se hace necesario implementar modelos correspondientes a los procesos educativos virtuales de excelente calidad y con ellos responder a la infraestructura técnica, educativa y regulativa que se ha ido postergando en algunos contextos educativos. Para tal efecto, uno de los retos más significativos que se deben abordar desde la educación virtual es la conectividad, lo cual exige una inversión presupuestal de gran magnitud buscando superar las brechas que se han generado debido a los contextos regionales y urbanos particulares.

Por parte del Gobierno Nacional y del Ministerio de Tecnologías de la Información y las Comunicaciones, se han implementado proyectos como Vive Digital y Aprender Digital, cuyo objetivo fundamental es llegar a los lugares más recónditos y vulnerables del país. Esta es una excelente iniciativa, sin embargo, apropiarse de las nuevas tecnologías como aporte mediático a los procesos de enseñanza aprendizaje y llevarlos a lugares apartados de la geografía nacional, resulta ser uno de los retos más grandes en cuanto al manejo presupuestal de los recursos financieros y la regulación de licencias y políticas públicas para lograr la cobertura territorial y virtual. Ahora bien, es importante el papel que cumplen las redes comunitarias locales y su infraestructura como posibilidad de conectividad a internet y que no están dentro del sistema operativo tradicional.

La conectividad implica apropiarse de modelos virtuales educativos adecuados según los contextos sociodemográficos, de tal manera que se modifiquen en esencia las prácticas de enseñanza $\mathrm{y}$ aprendizaje sin quedarse solamente en cambiar clases presenciales por clases remotas apoyadas en mediaciones y ambientes virtuales de aprendizaje de las TIC. La tecnología no debe ser solamente una herramienta de aprendizaje como las infraestructuras de software, debe ser una mediación para implementar calidad de vida educativa en la que los estudiantes, seres humanos, son el centro de atención. Por lo tanto, esta realidad implica considerar ritmos flexibilizados de enseñanza y aprendizaje contextualizados según las necesidades educativas de los estudiantes y los docentes y no estandarizados de manera análoga e igual para todos. Se necesita un trabajo colaborativo y de acompañamiento tanto para los encuentros sincrónicos que corresponden a las video llamadas, así como para los momentos asincrónicos donde se evidenciará más la atención disciplinada y autónoma de los estudiantes.

\section{Conclusiones}

Los procesos de modernización de la educación en Colombia cada vez más están mediados por la virtualidad, lo cual se convierte en una oportunidad para optimizar los procesos de enseñanza aprendizaje tanto personales como colectivos, en los diversos escenarios en los que las personas interactúan como los laborales, familiares y profesionales. La interactividad virtual permite aprendizajes significativos en orden a la cualificación profesional; contacto con nuevas culturas, nuevos idiomas, nuevas áreas y modelos de conocimiento. Desde la perspectiva de la modernización de la educación, los nuevos modelos de la aldea global han obligado a la educación a adquirir e implementar tecnologías que respondan a los procesos de alfabetización tecnológica $y$, por consiguiente, a considerar que el conocimiento tecnológico es una competencia fundamental para las nuevas generaciones profesionales (Suarez, 2014).

Se deben tener en cuenta aspectos fundamentales para que se pueda generar esta reforma educativa y dar paso a la "nueva era digital". Por lo tanto, es indispensable que unan fuerzas los dos pilares fundamentales que componen una nación: el Estado y la sociedad, los cuales deben tener un interés por generar desarrollo. Además, el Estado juega un papel importante como es la ayuda tanto económica y estructural buscando equidad social. Las TIC ofrecen amplias alternativas para mejorar la calidad de vida, pero depende de cada persona como les dé un buen uso y posterior aplicación. Igualmente, las TIC son un buen camino de aprendizaje ya que actualmente todo tipo de 
público puede acceder a ellas y enriquecer sus conocimientos.

La educación es un elemento fundamental para llevar a cabo el desarrollo de la sociedad y ha de enfrentar diversos retos como la implementación de las TIC. Estos nuevos retos no han de superar y trastocar los paradigmas culturales, sino ayudar a conservarlos como patrimonio social. Por lo tanto, las TIC responden a los nuevos paradigmas que experimenta la humanidad en su anhelo por conocer más y de manera eficiente, eficaz y efectiva. En este sentido, las TIC de la mano de la educación son perfectos aliados para el desarrollo de la sociedad

Aunque la educación virtual tiene muchas ventajas que favorecen a la población estudiantil, existen algunas fallas que se pueden ir mejorando para disminuir el acceso desigual entre las personas y así todos tengan la posibilidad de recibir educación conforme va avanzando la tecnología. Es necesario que todas las personas posean un acceso igualitario al uso de las tecnologías de la información, sin embargo, para que esto sea posible, se debe remitir a la responsabilidad que tiene el Estado para que: "mitigue las diferencias crecientes entre ciudadanos, grupos y regiones de cada país” (Paras, 2001, p.42) y esto se puede lograr con la implementación y regulación de políticas públicas facilitadoras de la cobertura y conectividad.

$\mathrm{El}$ acceso a las TIC da lugar al aval para pertenecer a la sociedad global tecnológica, lo cual implica romper las brechas digitales que se imponen en la sociedad educativa tradicional porque permite abrirse de manera flexible y adaptable a nuevos modelos y paradigmas comunicacionales en la adquisición del conocimiento. Por lo tanto, los recursos de la virtualidad aportan significativamente a la labor educativa en los procesos de formación integral e idónea de los profesionales y su disponibilidad de servicio a la sociedad y el desarrollo del país.

\section{Referencias}

Acevedo, A., y Villamizar, C. (2018). Discursos y prácticas culturales durante la "Revolución en Marcha". Reforma educativa y cambio social. Revista Ciencias Sociales y Educación, 4(7), 37-52. Recuperado de: https://revis-
tas.udem.edu.co/index.php/Ciencias_Sociales/article/ view/1562/1606

Balart, C., y Cortés, S. (2018). Una mirada histórica del impacto de las TIC en la sociedad del conocimiento en el contexto nacional actual. Contextos. Estudios de $\mathrm{Hu}$ manidades y Ciencias Sociales, 1(41), 2-19.

Barón, L. F., y Gómez, R. (2012). De la infraestructura a la apropiación social: panorama sobre las políticas de las tecnologías de información y comunicación (TIC) en Colombia. Signo y Pensamiento, 31(61), 38-71.

Bazdresch, M. (2001). Educación y pobreza: una relación conflictiva. En Pobreza, desigualdad social y ciudadanía (pp. 65-81). Buenos Aires, Argentina: CLACSO.

Becerra, G. (2017). La educación Virtual: Retos y desafíos en Colombia. Revista Empresarial y Laboral. Recuperado de https://revistaempresarial.com/educacion/ virtual/la-educacion-virtual-retos-desafios-colombia/

Contreras, M. E. (2018). La educación virtual se abre camino en Colombia. Universidad EAN. Recuperado de https:/universidadean.edu.co/noticias/la-educacion-virtual-se-abre-camino-en-colombia

Davila, S., (2006), Generación Net: visiones para su Educación Orbis. Revista Científica Ciencias Humanas, 1(3),24-48.

Facundo, Á. (2004). La virtualización desde la perspectiva de la modernización de la educación superior: consideraciones pedagógicas. Revista Universidad y Sociedad del Conocimiento, 1(1), 1-17. doi: https://doi. org/10.15765/plnt.vli2.353

Fajardo, E. (2019). Las nuevas tecnologías: ¿amenaza u oportunidad en el escenario educativo? Revista de la Facultad de Ciencias de la Educación, 4(1), 181-197.

González, J. N. (2001). Hacia una reforma educativa en la era digital. Revista Ibero Americana de Educación, (26).

González, M. C. (2017). Ventajas desventajas de la educación virtual. Palabra maestra. Recuperado de https:// www.compartirpalabramaestra.org/noticias/ventajas-y-desventajas-de-la-educacion-virtual

Herrera, A. (2015). Una mirada reflexiva sobre las TIC en Educación Superior. Revista Electrónica de Investigación Educativa, 17(1), 1-4.

Lugo, M. T., y Kelly, V. (2010). Tecnología en educación ¿Políticas para la innovación? IIPE-Unesco, Sede Regional Buenos Aires. Recuperad de http://sedici.unlp. edu.ar/bitstream/handle/10915/18441/Documento_ completo.pdf?sequence $=1 \&$ is Allowed $=y$

Maza, F., Vergara, J. y Román, R. (2017). Eficiencia y productividad en la cobertura de las universidades públicas colombianas. Revista Investigación y de- 
sarrollo, 25(2), 6-33, doi: https://doi.org/10.14482/indes.25.2.10957

Ministerio de Educación Nacional. (2017). Observatorio Laboral para la Educación. Recuperado de http://www. graduadoscolombia.edu.co/html/1732/w3-channel. html.

Munévar, D. (2017). Análisis de la política educativa colombiana desde la teoría del constructivismo estructuralista. Revista Boletín REDIPE, 6(2), 67-80.

Organización de Estados Iberoamericanos [OEI] (2001). Memoria-Programación 1999-2000. Madrid, Organización de Estados Iberoamericanos para la Educación, la Ciencia y la Cultura. Recuperado de https://www. oei.es/historico/memoria/memolo.htm

Paras, J. N. (2017). Hacia una reforma educativa en la era digital Revista Iberoamericana de Educación, (26). Recuperado de https://rieoei.org/historico/documentos/ rie26a04.htm
Santiago, R. M. (2001). Introducción. Revista Iberoamericana de Educación, (26). Recuperado de https://rieoei. org/historico/documentos/rie26a00.htm

Selwyn, N., (2013). Internet y educación. En C@mbio: 19 ensayos clave sobre cómo internet está cambiando nuestras vidas (pp. 191-254). Madrid, España: BBVA. Recuperado de https://www.bbvaopenmind. com/wp-content/uploads/2014/01/BBVA-OpenMind-libro-Cambio-19-ensayos-fundamentales-sobre-c\%C3\%B3mo-internet-est\%C3\%A1-cambiando-nuestras-vidas-Tecnolog\%C3\%ADa-Interent Innovaci\%C3\%B3n.pdf

Suárez, N. y Custodio, J. (2014). Evolución de las tecnologías de información y comunicación en el proceso de enseñanza-aprendizaje. Revista Vínculos, 11(1), 209-220.

Tapia, E. y León, J. (2013). Educación con TIC para la sociedad del conocimiento. Revista Digital Universitaria, 14(2). Recuperado de http://www.revista.unam.mx/ vol.14/num2/art16/ 